\title{
First Principles Approach to lonicity of Fragments
}

\author{
Ghanshyam Pilania, ${ }^{*}$ Xiang-Yang Liu, and Steven M. Valone \\ Materials Science and Technology Devision, Los Alamos National Laboratory, Los Alamos, \\ New Mexico 87545, USA \\ E-mail: gpilania@lanl.gov
}

\begin{abstract}
We develop a first principles approach towards the ionicity of fragments. In contrast to the bond ionicity, the fragment ionicity refers to an electronic property of the constituents of a larger system, which may vary from a single atom to a functional group or a unit cell to a crystal. The fragment ionicity is quantitatively defined in terms of the coefficients of contributing charge states in a superposition of valence configurations of the system. Utilizing the constrained density functional theory-based computations, a practical method to compute the fragment ionicity from valence electron charge densities, suitably decomposed according to the fragment Hamiltonian $(\mathrm{FH})$ model prescription for those electron densities, is presented for the first time. The adopted approach is illustrated using $\mathrm{BeO}, \mathrm{MgO}$ and $\mathrm{CaO}$ diatomic molecules as simple examples. The results are compared and discussed with respect to the bond ionicity scales of Phillips and Pauling.
\end{abstract}

${ }^{*}$ To whom correspondence should be addressed 


\section{Introduction}

Defining and characterizing the electronic states of atoms or functional groups (hereafter referred to as fragments) in molecules or materials has long been an important endeavor in chemical physics. Empirical concepts such as electronegativity, covalency, ionicity, valence charge and covalent radii are cases in point and therefore hold a unique place of importance when exploring a diverse set of materials belonging to a vast chemical space. These quantities not only facilitate a deeper intuitive understanding of a given problem, but also allow for studies aimed at efficient qualitative predictions, relative trend identifications and broad classifications of molecular or material systems. Furthermore, they serve as important descriptors in quantifying molecular similarity. ${ }^{1}$ Quantification of molecular similarity facilitates in identifying trends in chemical reactivity under the assumption that chemically similar molecules tend to exhibit comparable patterns of activity. Therefore, it is not surprising that these empirical features are frequently used in what have come to be called quantitative structure-property-relationship (QSPR) approaches. ${ }^{2,3}$

Among the empirical descriptors devised to train our intuition, the concept of ionicity has played a pivotal role in our current understanding of the nature of the chemical bond in both molecules and crystals. ${ }^{4}$ Concepts related to ionicity have been used in crystal structure classification of binary alloys, ${ }^{5-8}$ identification of phase-change materials, ${ }^{9}$ determination of surface atomic geometries,${ }^{10}$ quantifying molecular similarity in computational drug design. ${ }^{11}$ In the past, a number of different views and ideas have been put forward pertaining to the general definition of ionicity. Theories of Pauling based on thermochemical data, ${ }^{12}$ Coulson based on valence bond concepts ${ }^{13}$ and Phillips based on spectroscopic data ${ }^{14-16}$ are among the most prominent ones.

Pauling's definition of fractional ionic character $\tau^{P}$ for a chemical bond between $A$ and 
$B$ is based on the thermochemical arguments and is defined as,

$$
\tau^{P}=1-\exp \left(-\frac{E_{A B}^{f}-\frac{1}{2}\left\{E_{A A}^{f}+E_{B B}^{f}\right\}}{4 c}\right),
$$

where $E_{A B}^{f}, E_{A A}^{f}$ and $E_{B B}^{f}$ represent the formation energies of $A B, A A$ and $B B$ bonds and $c$ is a constant with dimensions of energy. The well known concept of Pauling electronegativity $(X)$ is based on the exponent in this equation in the sense that

$$
E_{A B}^{f}-\frac{1}{2}\left\{E_{A A}^{f}+E_{B B}^{f}\right\} \propto\left(X_{A}-X_{B}\right)^{2},
$$

where $X_{A}$ and $X_{B}$ are the electronegativities of $A$ and $B$, respectively. This energy difference appears explicitly in Pauling ionicity, so that $\tau^{P}=\tau^{P}(\Delta X)$ with $\Delta X=\left(X_{A}-X_{B}\right)$. Strictly speaking, $\tau^{P}$ in Eq. 1 is not guaranteed to be positive in all pairs of $A$ and $B$. By this token, it is not an occupation number, unless the energy difference is positive, as it will be for bonded pairs.

Several limitations apply to $\tau^{P}$. Clearly, the Pauling concept of ionicity when applied to homonuclear diatomics, where $\left(X_{A}-X_{B}\right)^{2}=0$, leads to $\tau^{P}=0$, regardless of the bond length. As will be shown later, this differs from the concept of fragment ionicity, which takes bond length dependent non-zero values for homonuclear diatomics. The concept of Pauling ionicity is also intended to apply to bonds near equilibrium. To see this limitation, consider the $A B$ bond stretched to dissociation. The energy difference on the left-hand side of Eq. 2 vanishes and $\tau^{P} \rightarrow 0$, for atoms in their ground states and regardless of their identity. This limiting value for $\tau^{P}$ does make intuitive sense: No ionic character remains for atoms in their ground state. The Pauling definition of ionicity can have a different value for $\tau^{P}$ depending whether the right- or left-hand side of Eq. (2) is used for nonequilibrium bond lengths.

Coulson's definition of ionicity $\tau^{C}$, on the other hand, is based on the molecular-orbital approach and the coefficients with a molecular orbital. $\tau^{C}$ for a tetrahedrally coordinated 
$s p^{3}$-hybridized octet binary compound $A B$ is given as

$$
\tau^{C}=\frac{\left|\lambda_{A}^{2}-\lambda_{B}^{2}\right|}{\lambda_{A}^{2}+\lambda_{B}^{2}},
$$

where $\lambda_{A}$ and $\lambda_{B}$ are the coefficients (determined variationally) of the atomic orbitals $\phi_{A}$ and $\phi_{B}$ centered on atoms $A$ and $B$, respectively, which form the molecular orbital $\psi=$ $\lambda_{A} \phi_{A}+\lambda_{B} \phi_{B}$, whether or not it is bonding, non-bonding, or anti-bonding. Because of this functional form, $\tau^{C}$ has the properties of an occupation number. Equation 3 holds under the assumption that the atomic wave functions are orthogonal.

As noted by Phillips, ${ }^{18}$ this approach gives about as good a definition of the ionicity as can be obtained in the context of a two-state model of a binary compound. However, the major shortcoming of this approach lies in the fact that it can only be applied to binary compounds, and not to ternary crystals for instance. If applied to homonuclear diatomics, then $\left|\lambda_{A}^{2}-\lambda_{B}^{2}\right|=0$ requiring that $\tau^{C}=0$, again irrespective of the bond length. At dissociation the coefficients may reach some non-zero values for diatomics. One expects the value of zero as discussed for $\tau^{P}$.

Phillips' dielectric model of ionicity relies on the spectroscopically obtained transition energies between bonding and anti-bonding states of $A^{N} B^{8-N}$ semiconductor crystals, with $N$ being the number of valance electrons. For a covalent crystal, the average energy gap $E_{g}$ between the bonding and anti-bonding states can be decomposed as a homopolar gap $E_{h}$ (produced by the symmetric part of the ionic potential) and a charge transfer gap $E_{c}$ (produced by the anti-symmetric part of the ionic potential) as

$$
E_{g}^{2}=E_{h}^{2}+E_{c}^{2}
$$

The fractional ionic-bond character $\tau^{P h}$,

$$
\tau^{P h}=\frac{E_{c}^{2}}{E_{h}^{2}+E_{c}^{2}},
$$


is defined as the Phillips ionicity. This definition also conforms to the properties of an occupation number. This microscopic dielectric theory based ionicity model provides a basis for understanding the structural trends, elastic properties, energy bands, optical properties, and thermochemistry of semiconductors. It has met tremendous success in its application to the classification of crystal structures of binary alloys. ${ }^{18,19}$ A limitation appears when one considers cases where the material becomes metallic (e.g. under compression). In those situations, $E_{g} \rightarrow 0$. Defining $\tau^{P h}$ becomes difficult. As dissociation of the crystal is approached, $E_{c}$ will go to zero because if its relationship with dielectric screening and therefore $\tau^{P h}$ does go to zero in that limit.

In addition to the above theoretical definitions of ionicity, Peng et $a .^{20}$ have presented a scheme to determine ionicity by using reflection high-energy electron diffraction (RHEED) and reflection electron microscopy. The degree of ionicity for $\mathrm{NiO}$ was deduced by matching experimental RHEED patterns obtained from an atomically flat surface with dynamical RHEED calculations employing scattering factors of neutral atoms (i.e. $\mathrm{Ni}$ or $\mathrm{O}$ ) and of their ions (i.e. $\mathrm{Ni}^{+2}$ or $\mathrm{O}^{-2}$ ) associated with the resulting distribution of charge around a particular lattice site in a crystal. Garcia and Cohen ${ }^{21}$ have introduced a first-principles ionicity scale based on the asymmetry of the charge-density distribution. A different bond ionicity scale has also been introduced based on the deviation of the centers of the maximally localized Wannier functions from the corresponding bond centers. ${ }^{22}$

From the above discussion, it seems preferable that electronic states of individual fragments of a molecule should determine ionicity. Furthermore, a reliable model to quantify ionicity should also allow for charge fluctuations between/among the individual fragments. This makes ionicity, at least theoretically, a many-electron property. Properties characterized by states of fragments must rely, by their very nature, on basic electronic structure theory. Today electronic structure theory methods allow us to study electronic structure and properties of a diverse range of molecules, solids, surfaces and interfaces ${ }^{23}$ and their constituent fragments. It provides an invaluable source of information for other levels of 
modeling, among those being atomistic ones. However, the bulk of the available electronic structure information comes from density-functional-theory (DFT) calculations that provide an energy, electronic density and orbital decomposition for the total system, not the individual fragments. The problem at hand is most often how to recover information about states of fragments from the available aggregate information.

To this end, we note that, while Coulson's definition of ionicity is based on Mulliken electronegativity, Mulliken himself is absent from this list of developers of a concept of ionicity. ${ }^{24}$ Recently though, such a concept of ionicity for fragments was advanced within the Fragment Hamiltonian (FH) approach, closely following Mulliken's approach to electronegativity. ${ }^{25-28}$ Fragment ionicity could be defined as an occupation number that is paired, in the sense of conjugate variables, with the chemical hardness of Parr and Pearson, ${ }^{29}$ in a manner analogous to how particle number and Mulliken electronegativity are conjugate pairs. ${ }^{30}$

In what follows, using diatomic molecules as examples, we demonstrate how to compute fragment charge and fragment ionicity directly from the valence charge distribution. The details of the FH approach are discussed in Section 2. By combining a constrained density functional theory (CDFT) approach with the FH model prescription for ionicity, we provide a first-principles recipe to compute the fragment charge $q$ and fragment ionicity $\tau$. Details of our method are provided in Section 3. Then, in Section 4, we discuss the results of the FH ionicity approach and compare them with bond ionicity scales. Finally, we summarize our results in a conclusion section.

\section{A Fragment-Hamiltonian Perspective of Ionicity}

Along the direction of descriptors of the states of atoms, the recently developed FH model, ${ }^{25-28}$ based on Moffitt's atoms-in-molecules (AIM) form of the many-electron Hamiltonian operator, ${ }^{31-35}$ puts forward a new perspective on ionicity as a variable paired with "fragment hardness". Within the broad framework of the FH model, a system can be expressed as a 
collection of fragments and the identity of the fragments can be defined as any collection of nuclei and electrons. Thus, a fragment may be any object from a single atom to a functional group or a unit cell to a molecule in a molecular fluid or crystal. It follows from AIM theory ${ }^{32}$ that any electronic Hamiltonian can be written as a sum of individual Hamiltonians $\hat{H}_{A}$ for each fragment $A$ in a system of interest and purely Coulombic pairwise interactions $\hat{V}_{A B}$ between fragment pairs $\mathrm{A}$ and $\mathrm{B}$. Here $\hat{H}_{A}$ is given by the sum of kinetic energy, electronnuclear, and electron-electron Coulomb operators for its electrons and nuclei. ${ }^{27}$ It is the same operator associated with that fragment in isolation, except one important distinction, to be discussed shortly. The Coulombic interactions in $\hat{V}_{A B}$ are the terms that come from electrons on $\mathrm{A}$ interacting with the nuclei and electrons of $\mathrm{B}$, as well as the electrons of $B$ interacting with the nuclei and electrons of A. These Coulombic terms account for all of the interaction operators not appearing in the Hamiltonians of the fragments themselves. Thus, the FH is simply the total electronic Hamiltonian rewritten as

$$
\hat{H}^{(\mathrm{f})} \equiv \sum_{A} \hat{H}_{A}+\frac{1}{2} \sum_{A B}{ }^{\prime} \hat{V}_{A B}
$$

$\sum^{\prime}$ means a double sum excluding terms with $B=A$.

In the case of isolated neutral fragments one always assigns a number of electrons equal to the nuclear charge for each fragment. However, in general, for interacting fragments there is no physical requirement for this assignment. In fact, $\mathrm{FH}$ does allow number of electrons on each fragment to vary and thereby captures electron itinerancy in the model. To further clarify this point, suppose a total system which is a diatomic molecule consisting of two atomic fragments, $A$ and $B$. The total Hamiltonian is $\hat{H}^{(\mathrm{f})}=\hat{H}_{A}+\hat{H}_{B}+\hat{V}_{A B}$. Assume that the system is neutral, so that $N=Z_{A}+Z_{B}$, where $N$ is the total number of electrons. $\hat{H}_{A}$ is the Hamiltonian for isolated A with $N_{A}$ electrons, and $\hat{H}_{B}$ is the Hamiltonian for isolated B with $N_{B}$ electrons. Note that, to maintain overall neutrality, it is only necessary that $N_{A}+N_{B}=N$. It is not required that $N_{A}=Z_{A}$. Therefore, $\hat{H}_{A}+\hat{H}_{B}=\hat{H}_{A}\left(Z_{A}-\zeta\right)+$ 
$\hat{H}_{B}\left(Z_{B}+\zeta\right)$ is implicit for any integer charge state $\zeta$ in the $\mathrm{FH}$ model. This also is the important distinction that was referred to in the previous paragraph.

Further note that, the FH model implements charge fluctuations at the level of the operators for each fragment. To explain this we take a concrete example of quantum mechanical particle charge density operator defined as $\hat{\rho}(\mathbf{r})=e \sum_{\mathbf{i}=\mathbf{1}}^{\mathbf{N}} \delta^{(\mathbf{3})}\left(\mathbf{r}-\mathbf{r}_{\mathbf{i}}\right)$, where the sum is over all electrons in the system. Its expectation value yields the particle charge density $\rho(\mathbf{r})$ for state $\Psi$

$$
\rho(\mathbf{r}) \equiv\left\langle\Psi\left\{\mathbf{r}_{i}\right\}|\hat{\rho}(\mathbf{r})| \Psi\left\{\mathbf{r}_{\mathbf{i}}\right\}\right\rangle,
$$

where the wave function $\Psi\left\{\mathbf{r}_{i}\right\}$ of the total many-electron system has an implicit dependence on the position $\mathbf{r}_{i}$ of the $i^{\text {th }}$ electron. In close analogy with the $\hat{H}^{(\mathrm{f})}$ of the total system, as described above, the total particle density operator $\hat{\rho}$ can also be decomposed into the particle density operators of the two fragments $\hat{\rho}_{A}$ and $\hat{\rho}_{B}$ as

$$
\hat{\rho}_{A}\left(N_{A}\right)=e \sum_{i=1}^{N_{A}} \delta^{(3)}\left(\mathbf{r}-\mathbf{r}_{i}\right)
$$

and

$$
\hat{\rho}_{B}\left(N_{B}\right)=e \sum_{i=N_{A}+1}^{N} \delta^{(3)}\left(\mathbf{r}-\mathbf{r}_{i}\right),
$$

where $N_{A}$ and $N_{B}$ are the integer numbers of electrons on $A$ and $B$, respectively. These numbers may or may not be equal to the corresponding nuclear charges. The difference between $N_{A}$ and $Z_{A}$ will be designated as the charge state $\zeta_{A}$ of the fragment $A$. We note for reference that similar reasoning about decomposition into operators for fragments also holds for kinetic energy and fragment-fragment Coulombic interaction operators. Therefore these fragment operators also depend explicitly on $\zeta_{A}$ and $\zeta_{B} \cdot{ }^{27}$ Since we are addressing diatomic molecules here, only one charge state variable $\zeta$ is necessary, because $\zeta_{B}=-\zeta_{A}$. 
Within the FH model, ${ }^{25-28}$ a trial state for an overall neutral diatomic molecule AB can be defined based on the allowed integer charge states of A and B as follows ${ }^{24,36,37}$

$$
|A B\rangle \equiv \sum_{\zeta} C_{\zeta}\left|A^{+\zeta} B^{-\zeta}\right\rangle
$$

where the sum runs over all the allowed charge states of $A$. The $C_{\zeta}$ are a set of coefficients that contain information about how much each state of $A B$ contributes to a trial wave function and therefore determine the occupation numbers for the various states. $\left|A^{+\zeta} B^{-\zeta}\right\rangle$ depicts the wave function describing the state of $\mathrm{AB}$ when $\mathrm{A}$ has integer charge $\zeta$.

At this point we stipulate that only three charge states will be allowed in our analyzes. Specifically, the three allowed charge state will be $-\zeta, 0$ and $+\zeta$. The allowed valence states are designated as $\left|A^{-\zeta} B^{+\zeta}\right\rangle,\left|A^{0} B^{0}\right\rangle$, and $\left|A^{+\zeta} B^{-\zeta}\right\rangle$.

It is important to recognize that, for $\zeta=1$, these three states are identical to those included by Mulliken in his definition of electronegativity. ${ }^{24}$ For this reason, there will be a strong connection between the FH definition of ionicity and Mulliken's work. One difference though is that our formulation of the definition will not be restricted to $\zeta=1$. For instance, for a bivalent diatomic molecule, $\zeta=2$ and the three charge states are $A^{+2} \mathrm{~B}^{-2}, \mathrm{~A}^{0} \mathrm{~B}^{0}$ and $\mathrm{A}^{-2} \mathrm{~B}^{+2}$. Even though only three charge states (and hence forth referred to as a three-state model) will be allowed in the following, this restriction is only for illustrative purpose and there is no formal restriction coming from the FH model itself.

In the present study, we only consider fragments that are individual atoms. For this reason, energies for the charge states of the atoms can be assembled into local values of chemical potential and optical gap (or chemical hardness). The mixing coefficients for these fragment energies map onto the well-known local, net charge on the atoms which is conjugate to the local chemical potential and the less-known, but still recognized, ionicity which turns out to be conjugate to the local optical gap.

Since the coefficients $C_{\zeta}$ represent the contributions of each state of AB to its total wave 


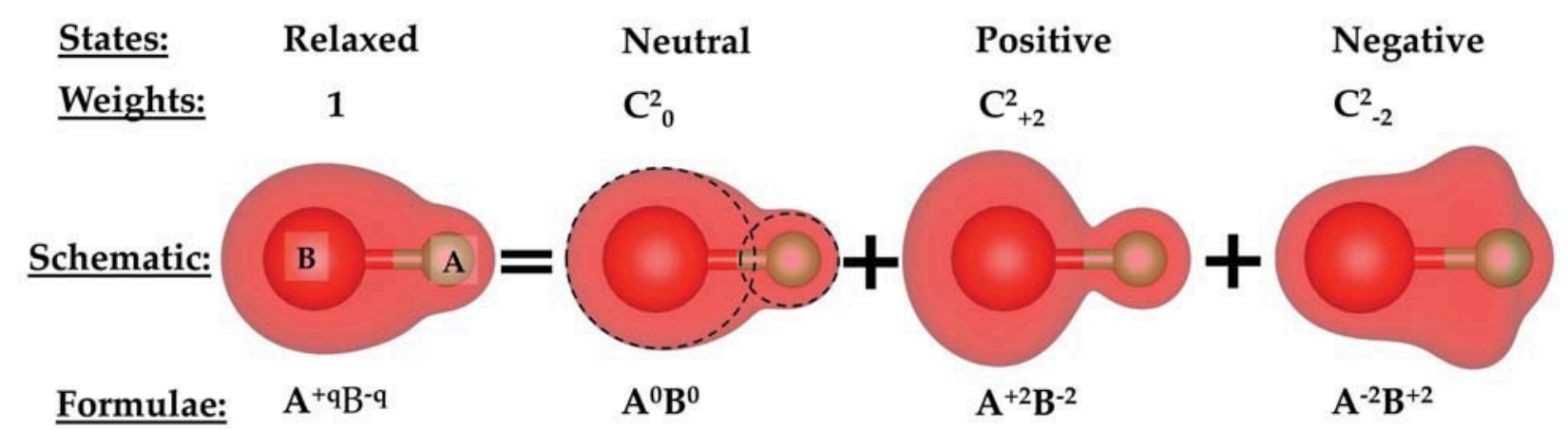

Figure 1: A schematic illustration of the three state $\mathrm{FH}$ model, where a relaxed charge density of a diatomic molecule $\mathrm{AB}$ can be represented as a weighted sum over the three pure integer charge states. The weight coefficients $C_{\zeta}^{2}$ are related to the charge and ionicity of the diatomic at a given bond length through Eqs. 11, 12 and 13.

function, they can be used to resurrect the real electron density associated with $A B$, which is

$$
\rho_{A B}=\frac{\sum_{\zeta} C_{\zeta}^{2} \rho_{A \zeta B^{-\zeta}}}{\sum_{\zeta} C_{\zeta}^{2}}
$$

where $\rho_{A{ }^{\zeta} B^{-\zeta}}$ represents the charge density associated with the state $A^{\zeta} B^{-\zeta}$. The transition densities between charge states are neglected as they are in most decompositions of electron densities. ${ }^{38-40}$ For the case of a bivalent diatomic molecule, within a three-state model as shown schematically in Figure 1, the above equation can be written as

$$
\rho_{A B}=\frac{C_{0}^{2} \rho_{A^{0} B^{0}}+C_{+2}^{2} \rho_{A^{+2} B^{-2}}+C_{-2}^{2} \rho_{A^{-2} B^{+2}}}{C_{0}^{2}+C_{+2}^{2}+C_{-2}^{2}}=\frac{\rho_{A^{0} B^{0}}+\lambda_{+}^{2} \rho_{A^{+2} B^{-2}}+\lambda_{-}^{2} \rho_{A^{-2} B^{+2}}}{1+\lambda_{+}^{2}+\lambda_{-}^{2}},
$$

where $\lambda_{ \pm}^{2}$ are defined as $C_{ \pm 2}^{2} / C_{0}^{2}$. Furthermore, fragment charge can then be defined as ${ }^{41}$

$$
q_{A}=-q_{B} \equiv \zeta \frac{\lambda_{+}^{2}-\lambda_{-}^{2}}{1+\lambda_{+}^{2}+\lambda_{-}^{2}},
$$

while our definition of fragment ionicity (or total ionic character) becomes

$$
\tau \equiv \frac{\lambda_{+}^{2}+\lambda_{-}^{2}}{1+\lambda_{+}^{2}+\lambda_{-}^{2}}
$$


To better balance these two definitions, we will define the scaled charge $q \equiv q_{A} / \zeta$. It can be seen that there is a strong conceptual relationship between the fragment and Coulson ionicities through their utilization of expansion coefficients, but better adherence with the dissociation limits of the Phillips ionicity. The metallic limits are different wherein $\tau \approx 0.5$ for good metals. Finally, $\tau$ meets the definition of an occupation number.

Further note that from the above two equations it follows that $\tau \geqslant|q|$. Additional insight into the value of the ionicity can be observed through Eq. 11, rewritten as a function of $q$ and $\tau . \rho_{A B}$ then becomes

$$
\rho_{A B}=\rho_{A^{0} B^{0}}+\frac{q}{2}\left(\rho_{A^{+2} B^{-2}}-\rho_{A^{-2} B^{+2}}\right)+\frac{\tau}{2}\left(\rho_{A^{+2} B^{-2}}+\rho_{A^{-2} B^{+2}}-2 \rho_{A^{0} B^{0}}\right) .
$$

In the present study, we have used CDFT computations to project the relaxed charge density of a diatomic molecule onto the charge densities of the three pure integer charge states to determine the occupation numbers for the pure states that contribute to the relaxed state. These occupation numbers are then used to compute the fragment charge $q$ and fragment ionicity $\tau$. Note that this is equivalent to using Eq. 14 directly with an additional constraint that $\tau \geqslant|q|$ to fit the charge density and obtain charge and ionicity directly without introducing occupation numbers. Further details of our method are provided in the next section.

\section{Methods}

The first crucial step towards computation of ionicity from first-principles within the $\mathrm{FH}$ prescription is construction of basis states of known integer charges and ionicities. Within Kohn-Sham DFT, ${ }^{43-46}$ it is possible to optimize the electronic charge density of a system with an arbitrary constraint on its electron density e.g., that there are $N$ charges on a

certain group of atoms. $\mathrm{Wu}$ and Voorhis ${ }^{47}$ have shown that the minimum energy state consistent with the given constraint on electronic charge density is a maximum with respect 
to the constrained potential, and that this solution is unique. Referred to as the constrained DFT implementation, CDFT computations were carried out using B3LYP ${ }^{48}$ (Becke's three parameter hybrid functional with the Lee-Yang-Parr correlation functional) with the 6-31G** level of basis sets as implemented in the NWChem package. ${ }^{49}$

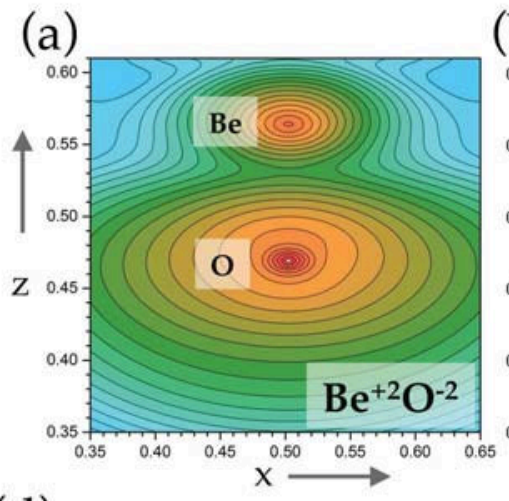

(d)

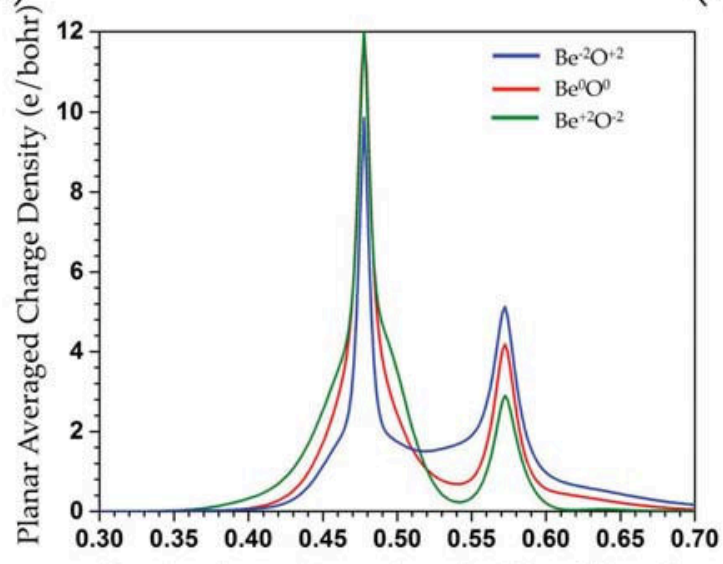

Fractional coordinate along Be-O bond length

(b)

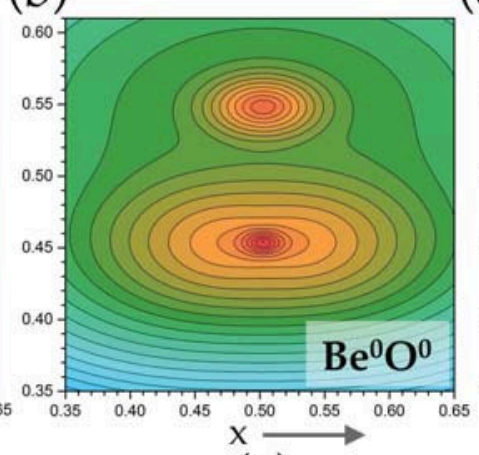

(c)

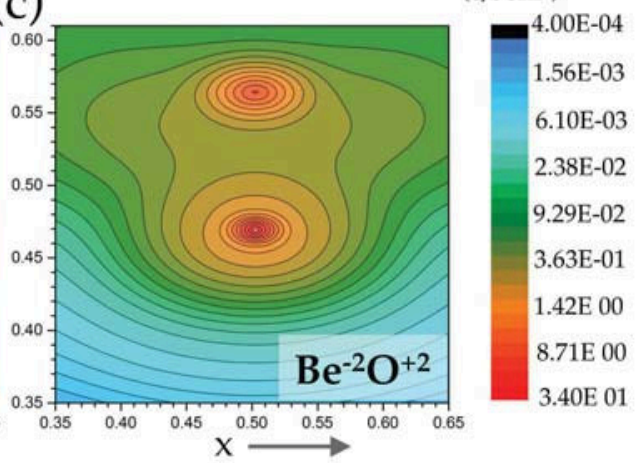

(e)

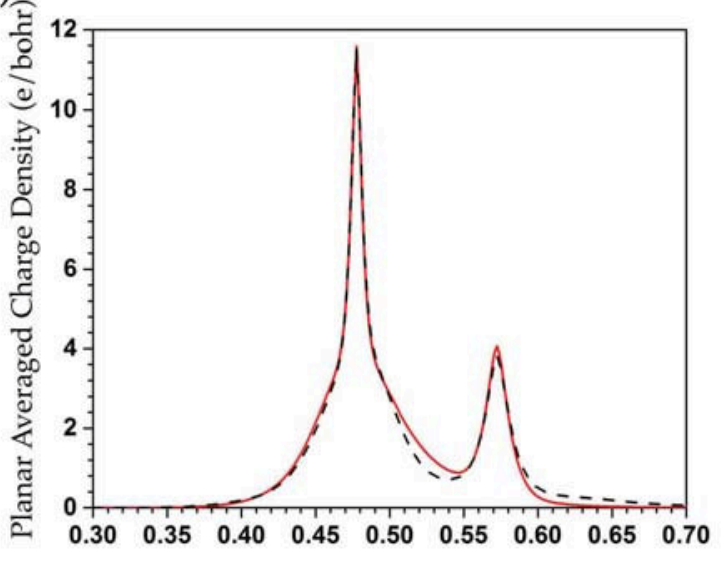

Fractional coordinate along Be-O bond length

Figure 2: Two-dimensional charge density contour plots for the $\mathrm{Be}-\mathrm{O}$ diatomic at the equilibrium bond length of $1.33 \AA$. (a) cationic $(\zeta=+2)$, (b) neutral $(\zeta=0)$ and (c) anionic $(\zeta=-2)$ charge states. (d) Planar averaged valence charge density profiles for the three $\mathrm{FH}$ basis states. These averages are taken in planes normal to the axis of the molecule. (e) A comparison between the DFT-computed and fitted (using Eq. 11) planar averaged valence charge density profiles.

In this study, we considered a three state model for the MO diatomic, where $\mathrm{M}=\mathrm{Be}$, $\mathrm{Mg}$ and $\mathrm{Ca}$ and $\mathrm{M}$ atom can adopt +2 , neutral and -2 charge states in the three distinct $\mathrm{FH}$ basis states (i.e., $\mathrm{M}^{+2} \mathrm{O}^{-2}, \mathrm{M}^{-2} \mathrm{O}^{+2}$ and $\mathrm{M}^{0} \mathrm{O}^{0}$ ). Since the part of the total valence charge associated with a given atom is not an observable, there is no unique way to decompose it 
into respective atomic contributions. In the present scheme, to quantify the charges of the three FH basis states, we have used the Bader charge definition, ${ }^{50,51}$ as it conforms best with the local view of spacial charge distribution adopted within the FH model. Note however that since CDFT (as implemented in the NWChem package) does not allow for a constrained relaxation directly with Bader charges, we constrained the Löwdin charges and systematically varied them in different CDFT runs and computed the corresponding Bader charges. ${ }^{51}$ For each diatomic molecule at a given bond length, this procedure was used to construct the positive and negative basis states with the known integer Bader charges. The same procedure was also employed to construct the non-integer charge states of all the diatomics studied in the present study, where Löwdin charges were varied in CDFT calculations first and Bader and fragment charges were computed afterwards.

Figure 2 (a) to (c) depict two-dimensional average charge density contour plots for the three integer charge states for a $\mathrm{BeO}$ diatomic at its equilibrium bond length of $1.33 \AA$. The positive and negative charge states for the diatomic are prepared using constrained DFT optimizations, while the neutral charge state is formed simply by a superposition of the atomic charge densities of $\mathrm{Be}$ and $\mathrm{O}$ atoms placed at $1.33 \AA$ apart (to prepare a reference state of zero charge and zero ionicity). ${ }^{52}$ Figure $2(\mathrm{~d})$ illustrates the planar averaged charge density profiles for the three reference states along the $\mathrm{Be}-\mathrm{O}$ bond axis direction. To determine the $q$ and $\tau$ within a three-state $\mathrm{FH}$ model, we used a least square fitting procedure to determine the coefficients in Eq. 11 which provide a best fit to the relaxed planar averaged charge density profile for the diatomic ( $c f$. Figure 2(e)) and subsequently lead to the $q$ and $\tau$. However, we note here that the Eq. 10 is only strictly true when employing a complete basis of charged states, and therefore the restrictions of the three charge state model ${ }^{24,36,37}$ in the present paper, although satisfactory, hinder a perfect reconstruction of the electronic density as can be seen from Figure 2(e). 


\section{Results and Discussion}

Including the FH model, most of the existing charge transfer models to compute site energies are built from the intuitive idea that an atom in a material can occupy certain integer charge states, most typically being the neutral, cation, and anion states. ${ }^{37}$ The charge resonances associated with these integer charge states play an important role when it comes to chemically accurate descriptions of the material. In any charge transfer model potential, one needs a prescription as to how the energy of each atom in the material changes with local charge fluctuations. Although numerous models have been constructed and reported in the literature, the principal model used for this purpose so far has been that of Iczkowski and Margrave (IM). Within the IM model, ${ }^{53}$ the energy of an atom $A$ with charge $q_{A}$ may be expressed as a quadratic polynomial, as follows

$$
E_{A}^{I M}(q)=E_{A}^{0}+\zeta \chi_{A} q+\eta_{A} q^{2}
$$

where $E_{A}^{0}$ is the energy of the neutral, isolated atom. $\chi_{A}$ and $\eta_{A}$ represent the absolute electronegativity and absolute hardness for the isolated atom $A$, respectively. It is clearly evident from the Eq. 15 that the implied chemical potential $\mu_{A}^{I M}$ in the IM model is linear in $q$ as given by $-d E_{A}^{I M} / d q=-\left(\zeta \chi_{A}+2 \eta_{A} q\right)$. Consequently, this model leads to an unimpeded charge flow when atoms dissociate and has been characterized as metallic in nature. ${ }^{54}$

Derived from first-principles based arguments, $\mathrm{FH}$ is a relatively recent model of the charge dependence of a fragment energy and chemical potential, that effectively addresses the aforementioned short comings of the IM model. The FH model smoothly transitions between approximately locally quadratic (in charge) at short bond lengths and piecewise linear behaviors at large nearest neighbor distances, contrasting the strictly quadratic behavior found in the IM model. Furthermore, the chemical potential model put forward by the FH formalism to regulate charge flow has been shown to be in close agreement with the analysis of Perdew et al (henceforth referred to as PPLB). ${ }^{55}$ While the net charge depen- 
(a)

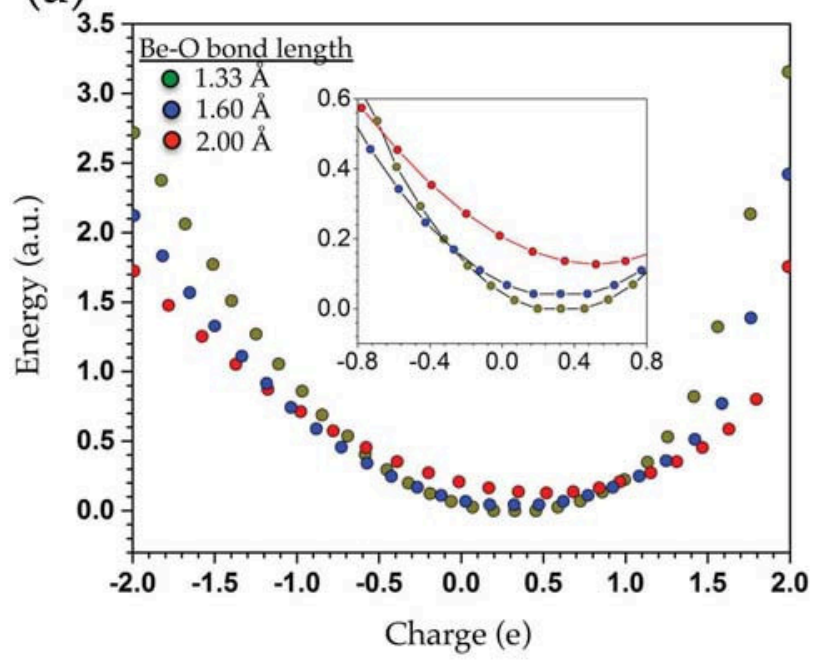

(b)

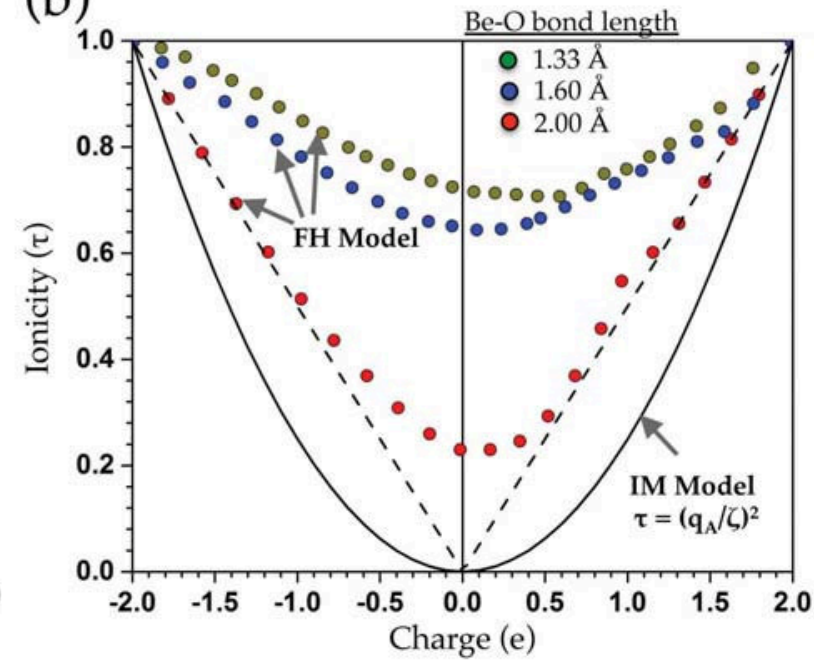

Figure 3: Variation of (a) total energy and (b) FH based ionicity $(\tau)$ as a function of fragment charge $\left(q_{A}\right.$, as defined in Eq. 12) for a $\mathrm{Be}-\mathrm{O}$ diatomic at three different bond lengths. The limiting case for the dissociation limit is shown by dashed lines in the panel (b). IM model based parabolic relationship is also shown using a solid black line.

dence associated with the electronegativity is the same in both the IM and FH models, the fragment chemical hardness appears as the coefficient of the total ionic character $\tau$ as ${ }^{27}$

$$
E_{A}^{F H}(q)=E_{A}^{0}+\zeta \chi_{A} q+\eta_{A} \tau(q)
$$

From comparison of Eqs. 15 and 16, it is clear that the IM approximation for $\tau(q)$ is always just $\tau(q)=\left(q_{A} / \zeta\right)^{2}=q^{2}$. Figures 3(a) and 3(b) show the variation of the total energy and the fragment ionicity as a function of the fragment charge for $\mathrm{BeO}$ diatomic for three different bond lengths. A theoretical limit of $\tau(q)$ is known and is important for atomistic models based on this notion to function properly. Regardless, of the diatomic or dimer, one always has at the dissociation limit a non-analytical relationship $\tau(q)=|q|$. This relationship recovers the PPLB limit of DFT for dissociated atoms, but in the context of a three-state model. ${ }^{56}$ Clearly, the dissociation limit is not satisfied by the IM model, as can be seen from Figure 3(b). Furthermore, this limit is also not attained by all DFT functionals. In other words, it is known that the local and many semi-local density functional approximations for 
the exchange-correlation energy can improperly dissociate a neutral molecule XY to fractionally charged fragments $\mathrm{X}^{+q}$ and $\mathrm{Y}^{-q}$ with an energy significantly lower than the neutral isolated atoms $\mathrm{X}^{0}$ and $\mathrm{Y}^{0}{ }^{57,58}$ The B3LYP functional does attain the proper dissociation limit, which is another reason for relying on this method to construct the atomically-based ionicity parameter. From 3(b), it can be seen that as the Be-O bond length is increased, the dissociation limit is systematically reached as the $\tau$ versus $q$ curve gradually approaches to the dashed line representing $\tau(q)=|q|$.

In the dissociation limit fragments of the diatomic behave as isolated atoms with a zero net charge and vanishing ionicity. The variation of $\tau$ as a function of $\mathrm{Be}-\mathrm{O}$ bond length is shown in Figure 4(a). Owing to the larger contributions from the anionic and cationic integer charge states, $\mathrm{BeO}$ diatomic shows a moderate $\tau$ near equilibrium and at shorter bond lengths. However, it quickly decays at larger bond lengths and shows a decaying tail behavior. It is clear from Figure 4(a) that the FH model indeed follows the correct dissociation limit.
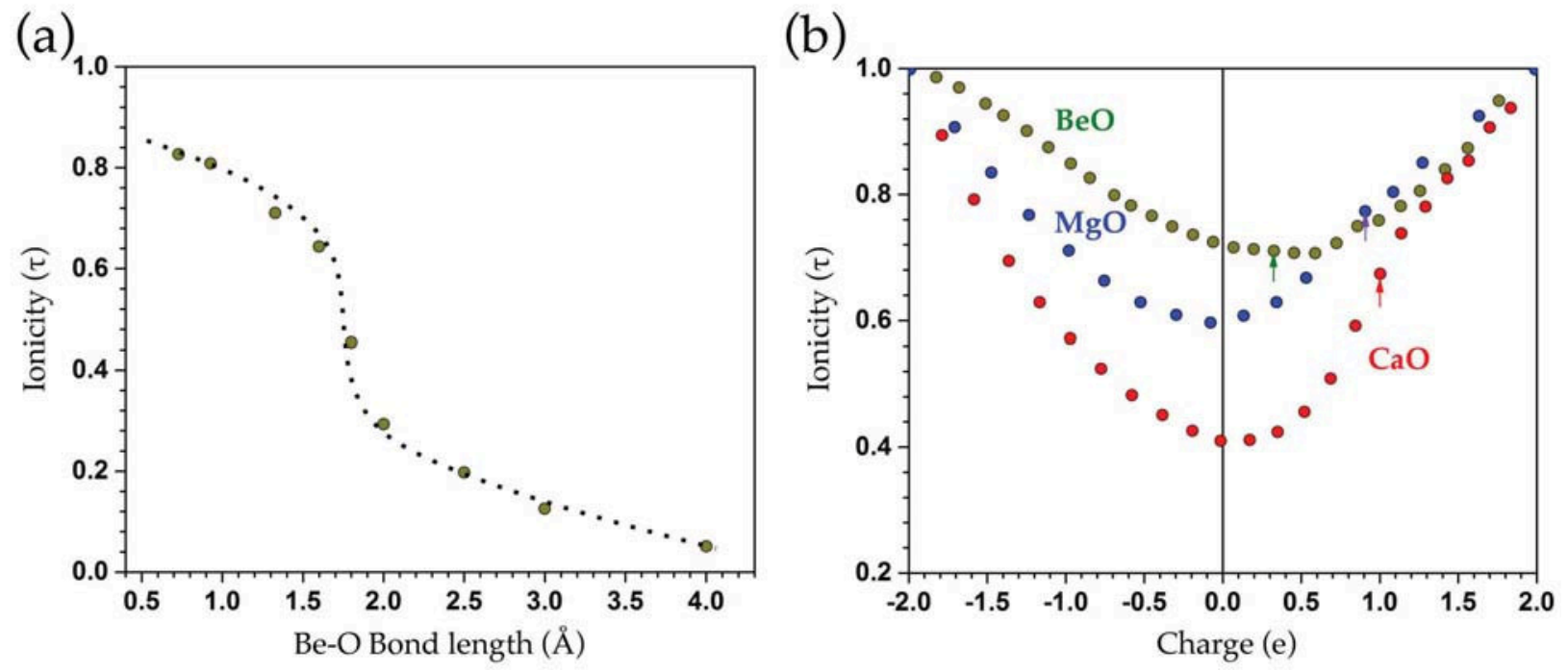

Figure 4: (a) Variation of the FH based ionicity as a function of the bond length for a Be- $\mathrm{O}$ diatomic. (b) fragment ionicity $(\tau)$ versus fragment charge $\left(q_{A}\right)$ plot for $\mathrm{Be}-\mathrm{O}, \mathrm{Mg}-\mathrm{O}$ and $\mathrm{Ca}-\mathrm{O}$ diatomics at their respective equilibrium bond lengths. The arrows mark the equilibrium points of lowest energy for each of the three cases. 
Table 1: Computed values of $\mathrm{FH}$ charges and Ionicities for $\mathrm{BeO}, \mathrm{MgO}$ and $\mathrm{CaO}$ diatomics. Corresponding available values of Phillips' and Pauling's Ionicities for solids are also included for comparison. ${ }^{4}$

\begin{tabular}{lcccc}
\hline \hline $\begin{array}{l}\text { Material } \\
\text { System }\end{array}$ & $\begin{array}{c}\text { FH Charge } \\
\left(q_{A}\right) \text { in e }\end{array}$ & $\begin{array}{c}\text { FH Ionicity } \\
(\tau)\end{array}$ & $\begin{array}{c}\text { Phillips } \\
\text { Ionicity }\left(\tau^{P h}\right)\end{array}$ & $\begin{array}{c}\text { Pauling } \\
\text { Ionicity }\left(\tau^{P}\right)\end{array}$ \\
\hline $\mathrm{BeO}$ & 0.326 & 0.710 & 0.602 & 0.63 \\
$\mathrm{MgO}$ & 0.908 & 0.773 & 0.841 & 0.73 \\
$\mathrm{CaO}$ & 1.291 & 0.780 & 0.913 & 0.79 \\
\hline
\end{tabular}

To see how well chemical trends are captured within the present $\mathrm{FH}$ based prescription of $\tau$, next we compute and compare $\tau$ values of $\mathrm{BeO}, \mathrm{MgO}$ and $\mathrm{CaO}$ diatomics. Figure 4(b) shows the results for all possible values of $\mathrm{FH}$ charges for the three systems at their respective equilibrium bond lengths. It can be seen that for $q= \pm 1$, we always get $\tau=1$, since the diatomic completely overlaps with one of its integer charge state (anionic or cationic) of unit ionicity. We further note that at the limit of vanishing charge (i.e., $q=0$ ) all three systems show a nonzero ionicity; this indicates the fact that the zero charge ground states for each of the three diatomics derives non-zero but equal (to result in a net zero charge) contributions from their respective cationic and anionic integer charge states. The data points representing optimal FH $q$ and $\tau$ for each of the three diatomics are highlighted using arrows in Figure 4(b) and the corresponding values are provided in Table 1. In the Table 1 we have also included available values of Phillips' and Pauling's ionicities for the three systems. ${ }^{4}$ Although a quantitative comparison is not possible because the literature values of ionicities reported here are for solids, nevertheless our computed values for the diatomics show a qualitative agreement with correct chemical trends.

Next, we take a look at the fragment charges computed at three different bond lengths for a $\mathrm{BeO}$ diatomic and compare them with other three charge definitions frequently used in literature, namely, those of Mulliken, ${ }^{59-62}$ Bader $^{63,64}$ and Löwdin. ${ }^{65}$ Figure 5 (a) to (c) show our computed Mulliken, Bader and FH charges plotted against Löwdin charges for a BeO diatomic at three different bond lengths (viz., 1.33, 1.60 and $2.00 \AA$, respectively). Our 

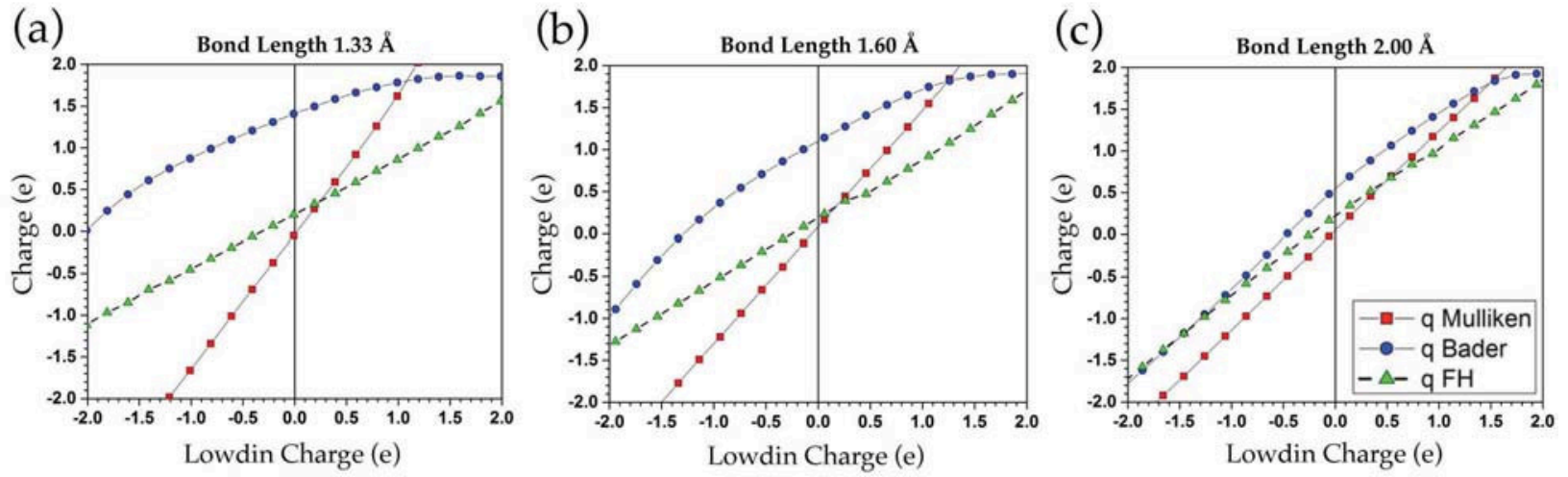

Figure 5: Variation of Mulliken, Bader and $\mathrm{FH}$ charges against Löwdin charges for a $\mathrm{BeO}$ diatomic plotted at a bond length of (a) $1.33 \AA$, (b) $1.60 \AA$ and (c) $2.00 \AA$.

results reveal that for a fixed bond length $\mathrm{FH}$ charges show an approximate linear trend with Löwdin charges, similar to that exhibited by Mulliken charges albeit with a different slope. On the other hand, Bader charges deviate significantly from any of the other three charge definition and show a non-linear trend. We further note that this deviation is readily evident at relatively small bond lengths ( $c f$., Figure 5(a)), which are comparable to the equilibrium bond length. However, for the larger bond lengths ( $\gtrsim 2.00 \AA$ ), all the four charge definitions (i.e., Mulliken, Bader, Löwdin and FH charges) gradually converge, as can be seen from Figure 5(c).

With these results in hand, we return to the relationship between fragment ionicity and the approach to electronegativity taken by Mulliken. ${ }^{24}$ To make the comparisons more direct, we consider the case where $\zeta=+1$ in the definition of the valence states. Next, note that Mulliken was principally relied on a diatomic $\mathrm{AB}$ at the dissociation limit, where coulombic interactions vanish. Then the total energy for this state can be written as

$$
E_{A B}=E_{A^{0}}+E_{B^{0}}+\left(\chi_{A}-\chi_{B}\right) q+\left(\eta_{A}+\eta_{B}\right) \tau
$$

where $q$ and $\tau$ are defined as in Eqs. 12 and 13, but with $\zeta=+1$, and $\eta_{A}$ and $\eta_{B}$ are the fragment chemical hardnesses. Thus, carried to its logical conclusions, Mulliken might well 
have arrived at an ionicity in this manner. Note too that the states that Mulliken operated with could not be categorized as originating from either molecular orbital or simple valence bond states. Furthermore, the electronegativity-difference term vanishes if $A$ and $B$ are the same atom types. Then, to get the correct ground state at the dissociation limit, it would be necessary to determine that $\tau=0$. Given that chemical hardnesses are positive for all elements in the periodic table, a nonzero $\tau$ could only raise the energy of the diatomic.

Finally, we note that the first-principles scheme of quantifying charge and ionicity within the $\mathrm{FH}$ view of materials provides a possibility for capturing all three major classes of bonding, namely covalent, metallic and ionic, in a single, unified model. In general, covalent materials are defined as those that predominantly occupy the neutral state and therefore show both low net charge and ionicity. In ionic materials, on the other hand, either cationic or anionic states dominate the ground state, leading to a high net charge as well as high ionicity. Metallic materials occupy a state with zero net charge, but still possess moderate ionicity because of the charge fluctuations in the system owing to the enhanced electron hopping. Contributions from cationic and anionic states are significant but comparable in magnitude, leading to a vanishing net charge. Therefore, metallic bonding within the context of embedded atom methods is a subject of primary interest for the $\mathrm{FH}$ approach for a deeper understanding of different components of the embedding energy. ${ }^{25}$ Furthermore, owing to its quantum mechanical origin and direct dependence on the electron density, the FH ionicity might be used to devise both global and local reactivity descriptors to predict chemical behavior of molecular species within the QSPR formalism. ${ }^{66}$

\section{Conclusions}

A first-principles scheme for quantifying charge and ionicity within the $\mathrm{FH}$ approach ${ }^{25-28}$ is presented. Distinct from bond ionicities, the fragment ionicity defined here corresponds to a constituent fragment of a larger system, which can in principle be any entity from a single 
atom to a functional group or a unit cell in a crystal. The fragment definition of ionicity extends both the concept to a larger variety of interacting fragment pairs than diatomics, the range of bond lengths for which the concept remains valid, and yields non-zero ionicities for dimers. Constrained density functional theory-based computations have been employed to devise a practical recipe to compute the ionicity from valence electron charge density decomposition for several diatomic molecules. In the context of the same three-state model employed by Mulliken and others, ${ }^{24,36,37}$ it is shown that the dissociation limit required to regulate charge flow is systematically reached as the FH based ionicity systematically vanishes at large bond lengths while obeying the theoretical limit $\tau(q)=|q|$. As the fragment charges are allowed to fluctuate on a diatomic molecule, they are shown to vary approximately linear with Löwdin charges, quite similar to that behavior exhibited by Mulliken charges. These fragment charges and ionicities described here will serve as input parameters for a new class of many-body potential energy function for atomistic modeling capable of capturing metallic, covalent and ionic bonding environments on an equal footing.

Acknowledgements: This work was supported by the Los Alamos National Laboratory (LANL) Directed Research and Development Program (20120053ER). LANL is operated by Los Alamos National Security, LLC, for the National Nuclear Security Administration of the U.S. Department of Energy under Contract No. DE-AC52-06NA25396.

\section{References}

(1) A. Bender and R. C. Glen, Org. Biomol. Chem., 2, 3204 (2004).

(2) T. Le, V. C. Epa, F. R. Burden and D. A. Winkler, Chem. Rev. 112, 2889 (2012).

(3) A. R. Katritzky, V. S. Lobanov and M. Karelson, Chem. Soc. Rev. 24, 279 (1995).

(4) J. C. Phillips, Rev. Mod. Phys. 42, 317 (1970).

(5) J. St. John and A. N. Bloch, Phys. Rev. Lett. 33, 1095 (1974). 
(6) J. R. Chelikowsky and J. C. Phillips, Phys. Rev. B 17, 2453 (1978).

(7) A. Zunger, Phys. Rev. Lett. 44, 582 (1980).

(8) W. Andreoni, G. Galli, Phys. Chem. of Miner. 14, 389 (1987).

(9) D. Lencer, M. Salinga, B. Grabowski, T. Hicke, J. Neugebauer and M. Wuttig, Nature Mater. 7, 972 (2008).

(10) J. E. Klepeis, C. Mailhiot, M. van Schilfgaarde and M. Methfessel, J. Vac. Sci. Technol. B 11, 1463 (1993).

(11) R. Carbo-Dorca ed. Molecular quantum similarity in QSAR and drug design. Vol. 73. Springer, 2000.

(12) L. Pauling, The Nature of the Chemical Bond (Cornell University Press, New York, 1960).

(13) C. A. Coulson, L. B. Redei, and D. Stocker, Proc. Roy. Soc. (London) 270, 357 (1962).

(14) J. C. Phillips, Physics Today 23, 23 (1970).

(15) J. C. Phillips, Phys. Rev. Lett. 20, 550 (1968).

(16) J. C. Phillips, Phys. Rev. Lett. 22, 645 (1969).

(17) E. P. Gyftopoulos and G. N. Hastopoulos, Proc Nat Acad Sci USA 60, 786 (1968).

(18) J. C. Phillips, Bonds and Bands in Semiconductors (Academic Press, New York, 1973).

(19) J. R. Chelikowsky and J. K. Burdett, Phys. Rev. Lett. 56, 961 (1986).

(20) L. M. Peng, S. L. Dudarev, and M. J. Whelan, Phys. Rev. B 56, 15314 (1997).

(21) A. Garcia and M. L. Cohen, Phys. Rev. B 47, 4215 (1993).

(22) H. Abu-Farsakh and A. Qteish, Phys. Rev. B 75, 085201 (2007). 
(23) R. M. Martin, Electronic Structure: Basic Theory and Practical Methods (Cambridge University Press, New York, 2004).

(24) R. S. Mulliken, J. Chem. Phys. 2, 782 (1934).

(25) S. M. Valone, S. R. Atlas and M. I. Baskes, Modelling Simul. Mater. Sci. Eng. 22 045013 (2014).

(26) S. M. Valone, J. Phys. Chem. Lett. 2, 2618 (2011).

(27) S. M. Valone, J. Chem. Theory Comput. 7, 2253 (2011).

(28) S. M. Valone and S. R. Atlas, J. Chem. Phys. 120, 7262 (2004).

(29) R. G. Parr and R. G. Pearson, J. Am. Chem. Soc. 105, 7512 (1983).

(30) P. Attard, Thermodynamics and statistical mechanics: equilibrium by entropy maximisation (Academic Press, 2002).

(31) W. Moffitt, Proc. Roy. Soc. A 210, 224 (1951).

(32) W. Moffitt, Proc. Roy. Soc. A 210, 245 (1951).

(33) W. Moffitt, Proc. Roy. Soc. A 218, 486 (1953).

(34) W. Moffitt and Scanlan, J. Proc. Roy. Soc. A 218, 464 (1953).

(35) W. Moffitt, Rep. Progr. Phys. 17, 173 (1954).

(36) J. C. Slater, Phys. Rev. 35, 509 (1930).

(37) L. Pauling, J. Am. Chem. Soc. 54, 3570 (1932).

(38) F. L. Hirshfeld, Theor. Chim. Acta 44, 129 (1977).

(39) A. Cedillo, P. K. Chattaraj, R. G. Parr, Int. J. Quant. Chem. 77, 403 (2000). 
(40) R. F. Nalewajski and R. G. Parr, Proc. Natl. Acad. Sci. 97, 8879 (2000).

(41) J. Morales, and T. J. Martínez, J. Phys. Chem. A 105, 2842 (2001).

(42) P. W. Ayers and R. G. Parr, J. Am. Chem. Soc. 122, 2010 (2000).

(43) W. Kohn, Rev. Mod. Phys. 71, 1253 (1999).

(44) P. Hohenberg and W. Kohn, Phys. Rev. 136, B864 (1964).

(45) W. Kohn and L. J. Sham, Phys. Rev. A 140, 1133 (1965).

(46) J. P. Perdew, K. Burke, and M. Ernzerhof, Phys. Rev. Lett. 77, 3865 (1996).

(47) Q. Wu and T. Van Voorhis, Phys. Rev. A 72, 024502 (2005).

(48) A. D. Becke, J. Chem. Phys. 98, 5648 (1993).

(49) M. Valiev, E. J. Bylaska, N. Govind, K. Kowalski, T. P. Straatsma, H. J. J. van Dam, D. Wang, J. Nieplocha, E. Apra, T. L. Windus and W. A. de Jong, Comput. Phys. Commun. 181, 1477 (2010).

(50) R. F. W. Bader, Atoms in molecules: a quantum theory (Oxford University Press, Oxford, 1990).

(51) W. Tang, E. Sanville, and G. Henkelman, J. Phys.: Condens. Matter 21, 084204 (2009).

(52) The neutral charge state was deliberately prepared by a superposition of the atomic charge densities (unlike the cationic and anionic basis states where a constrained relaxation was allowed) to prevent mixing of the neutral basis state with the charged basis states and thereby making sure that the neutral state remains purely covalent (as is the case for infinitely separated neutral atomic species).

(53) R. P. Iczkowski, J. L. Margrave, J. Am. Chem. Soc. 83, 3547 (1961). 
(54) H. S. Smalø, P. -O. Åstrand, and L. Jensen, J. Chem. Phys. 131, 044101 (2009).

(55) J. P. Perdew, R. G. Parr, M. Levy, J. L. Balduz, Phys. Rev. Lett. 49, 1691 (1982).

(56) S. M. Valone, J. Li, S. Jindal, Int. J. Quantum Chem. 108, 1452 (2008).

(57) A. Ruzsinszky, J. P. Perdew, G. I. Csonka, O. A. Vydrov, and G. E. Scuseria, J. Chem. Phys. 125194112 (2006).

(58) J. P. Perdew, in Density Functional Methods in Physics, pages 265-308, eds. R.M. Dreizler and J. da Providencia (Plenum, 1985).

(59) R. S. Mulliken, J. Chem. Phys. 23, 1833 (1955).

(60) R. S. Mulliken, J. Chem. Phys. 23, 1841 (1955).

(61) R. S. Mulliken, J. Chem. Phys. 23, 2339 (1955).

(62) R. S. Mulliken, J. Chem. Phys. 23, 2343 (1955).

(63) R. F. W. Bader, P. J. MacDougall, and C. D. H. Lau, J. Am. Chem. Soc. 106, 1594 (1984).

(64) W. Tang, E. Sanville, and G. Henkelman, J. Phys.: Condens. Matter 21, 084204 (2009).

(65) P. Löwdin, J. Chem. Phys. 18, 365 (1950).

(66) N. Sukumar, A Matter of Density: Exploring the Electron Density Concept in the Chemical, Biological, and Materials Sciences (John Wiley \& Sons Inc., New Jersey, 2012). 


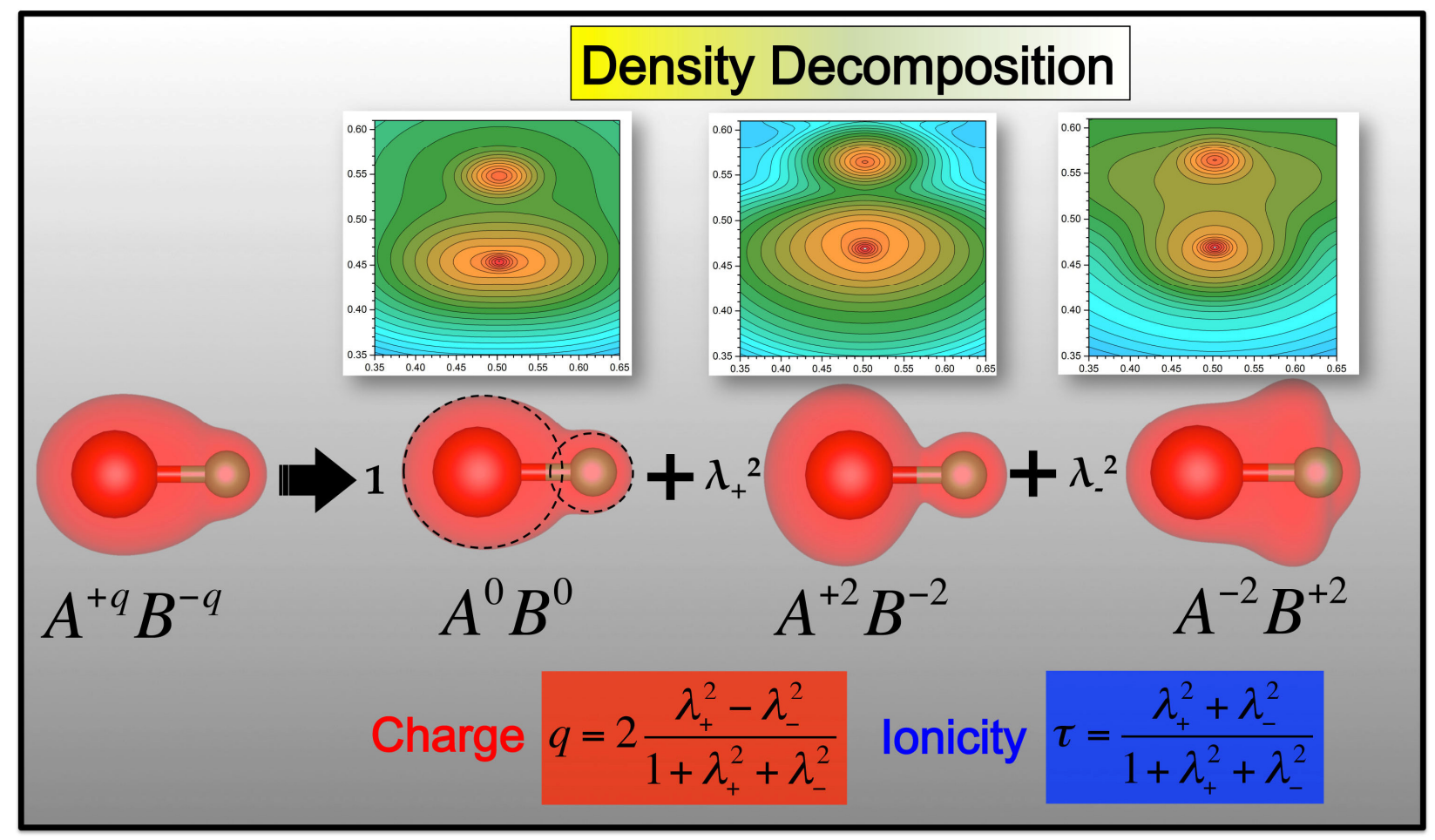

